\title{
Some New Results about The Period of Recurring Decimal
}

\author{
Bo Zhang \\ Linyi University at Feixian \\ Feixian, Shandong, P.R.China
}

\begin{abstract}
This study mainly discusses period problem of recurring decimals.According to Euler theorem, this paper gives the computation formula of period of recurring decimal, relation of the period and least positive period, and the necessary and sufficient condition that the period is eaqual to least positive period.
\end{abstract}

Keywords-Recurring decimal;Period;Prime.

\section{INTRODUCTION}

We know, irreducible proper fraction can be transformed recurring decimal (pure recurring decimal or mixed recurring decimal), the repetend digit of the recurring decimal is called the period of the recurring decimal, the least repetend digit is called least positive period.

Period problem of the recurring decimal is always a very interesting and difficult problem in number theory; many scholars studied it and derived some beautiful properties of the recurring decimals [1, 2, 5-10]. However, for the period and least positive period of the recurring decimal, there are not computation formulas so far.

Let us now consider 50 fractions between $1 / 3$ and $1 / 62$ (besides the fractions which can be denoted as limited decimals), and further convert them into recurring decimals, then their least positive period are as follows.

TABLE 1. Fractions AND LEAST Positive PERIODS

\begin{tabular}{|l|c|l|c|}
\hline \multicolumn{1}{|c|}{ Fraction } & $\begin{array}{c}\text { Least positive } \\
\text { period }\end{array}$ & \multicolumn{1}{|c|}{ Fraction } & $\begin{array}{c}\text { Least positive } \\
\text { period }\end{array}$ \\
\hline $1 / 3$ & 1 & $1 / 36=1 / 4 * 1 / 9$ & 1 \\
\hline $1 / 6=1 / 2 * 1 / 3$ & 1 & $1 / 37$ & 3 \\
\hline $1 / 7$ & 6 & $1 / 38=1 / 2 * 1 / 19$ & 18 \\
\hline $1 / 9$ & 1 & $1 / 39=1 / 3 * 1 / 13$ & 6 \\
\hline $1 / 11$ & 2 & $1 / 41$ & 5 \\
\hline $1 / 12=1 / 3 * 1 / 4$ & 1 & $1 / 42=1 / 6^{*} 1 / 7$ & 6 \\
\hline $1 / 13$ & 6 & $1 / 43$ & 21 \\
\hline $1 / 14=1 / 2 * 1 / 7$ & 6 & $1 / 44=1 / 4 * 1 / 11$ & 2 \\
\hline $1 / 15=1 / 5 * 1 / 3$ & 1 & $1 / 45=1 / 5 * 1 / 9$ & 1 \\
\hline $1 / 17$ & 16 & $1 / 46=1 / 2 * 1 / 23$ & 22 \\
\hline $1 / 18=1 / 2 * 1 / 9$ & 1 & $1 / 47$ & 46 \\
\hline $1 / 19$ & 18 & $1 / 48=1 / 16^{*} 1 / 3$ & 1 \\
\hline $1 / 21=1 / 3 * 1 / 7$ & 6 & $1 / 49=1 / 7 * 1 / 7$ & 42 \\
\hline $1 / 22=1 / 2 * 1 / 11$ & 2 & $1 / 51=1 / 3 * 1 / 17$ & 16 \\
\hline $1 / 23$ & 22 & $1 / 52=1 / 4 * 1 / 13$ & 6 \\
\hline $1 / 24=1 / 8 * 1 / 3$ & 1 & $1 / 53$ & 13 \\
\hline $1 / 26=1 / 2 * 1 / 13$ & 6 & $1 / 54=1 / 6 * 1 / 9$ & 3 \\
\hline $1 / 27$ & 3 & $1 / 55=1 / 5 * 1 / 11$ & 2 \\
\hline $1 / 28=1 / 4 * 1 / 7$ & 6 & $1 / 56=1 / 8^{*} 1 / 7$ & 6 \\
\hline
\end{tabular}

\begin{tabular}{|l|c|l|c|}
\hline $1 / 29$ & 28 & $1 / 57=1 / 3 * 1 / 19$ & 18 \\
\hline $1 / 30=1 / 10 * 1 / 3$ & 1 & $1 / 58=1 / 2 * 1 / 29$ & 28 \\
\hline $1 / 31$ & 15 & $1 / 59$ & 58 \\
\hline $1 / 33=1 / 3 * 1 / 11$ & 2 & $1 / 60=1 / 20 * 1 / 3$ & 1 \\
\hline $1 / 34=1 / 2 * 1 / 17$ & 16 & $1 / 61$ & 60 \\
\hline $1 / 35=1 / 5 * 1 / 7$ & 6 & $1 / 62=1 / 2 * 1 / 31$ & 15 \\
\hline
\end{tabular}

By observing Table 1, we find that their least positive periods have not distinct regularity. According to Euler theorem, we will present the computation formula of period of recurring decimal, relation of the period and least positive period, and the necessary and sufficient condition that the period is eaqual to least positive period.

\section{PRELIMINARIES}

Definition 1 Let $a / b$ be an irreducible proper fraction, when it can be denoted as a recurring decimal, the repetend digit of the recurring decimal(including pure recurring decimal and mixed recurring decimal) is called the period of $a / b$, and it is denoted by $T(a / b)$; the least repetend digit is called least positive period of $a / b$, and it is denoted by $\underline{T}(a / b)$.

Lemma 1[3] Let $a / b$ be an irreducible proper fraction, where

$$
b=2^{s} 5^{t}, s \geq 0, t \geq 0, s+t>0,
$$

then $a / b$ can be denoted as a limited decimal.

Lemma 2[3] Suppose that $a / b$ is an irreducible proper fraction, and $(b, 10)=1$, then

(i) $a / b$ can be denoted as a pure recurring decimal.

(ii) If $n_{0}$ is least positive integer such that

$$
10^{n} \equiv 1(\bmod b),
$$

then $\underline{T}(a / b)=n_{0}$.

Lemma 3[3] Suppose that $a / b$ is an irreducible proper fraction,

where 


$$
b=2^{s} 5^{t} b_{1},\left(b_{1}, 10\right)=1, s \geq 0, t \geq 0, s+t>0,
$$

$h=\max \{s, t\}$, then

(i) $a / b$ can be denoted as a mixed recurring decimal.

(ii) The digit of noncyclic part is $h$ in the decimal part of mixed recurring decimal.

(iii) If $n_{0}$ is least positive integer such that

$$
10^{n} \equiv 1\left(\bmod b_{1}\right)
$$

then $\underline{T}(a / b)=n_{0}$.

\section{ThE MAIN Results}

Theorem 1 Assume that $a / b$ is an irreducible proper fraction, $b=\prod_{i=1}^{n} p_{i}^{k_{i}}, k_{i} \geq 1,\left(p_{i}, 10\right)=1$, and $p_{i}$ is prime, then we have that

(i) $T(a / b)=\varphi(b)=\prod_{i=1}^{n} p_{i}^{k_{i}-1}\left(p_{i}-1\right)$ and $\underline{T}(a / b) \mid T(a / b)$;

(ii) $\underline{T}(a / b)=T(a / b)$ if and only if

$10^{i} \not \equiv 10^{j}(\bmod b)$, for any $i, j(0 \leq i<j \leq \varphi(b)-1)$.

Proof. (i) Since $k_{i} \geq 1,\left(p_{i}, 10\right)=1$, and $p_{i}$ is prime, thus

$$
\left(p_{i}^{k_{i}}, 10\right)=1 \text {, i.e. }(b, 10)=1 .
$$

From Euler theorem[4], we have that

$$
10^{\varphi(b)} \equiv 1(\bmod b) \text {, i.e. } b \mid\left(10^{\varphi(b)}-1\right) .
$$

Let $10^{\varphi(b)}-1=b k$ ( $k$ denotes integer), we know from $a<b$ that $a k<b k$, hence we can set

$$
a k=\overline{a_{1} a_{2} \mathrm{~L} a_{\varphi(b)}}\left(0 \leq a_{i} \leq 9\right),
$$

where $\overline{a_{1} a_{2} \mathrm{~L} a_{\varphi(b)}}$ denotes the positive integer composed of $a_{1}, a_{2}, \mathrm{~L}, a_{\varphi(b)}$.

Note that

$$
\begin{aligned}
& \left(10^{\varphi(b)}-1\right) \bar{\bullet} \cdot \stackrel{\bullet}{a_{1} a_{2} \mathrm{~L} a_{\varphi(b)}^{\bullet}} \\
& =\overline{a_{1} a_{2} \mathrm{~L} a_{\varphi(b)} \cdot \dot{a}_{1} a_{2} \mathrm{~L} a_{\varphi(b)}^{\bullet}}-\overline{0 . a_{1} a_{2} \mathrm{~L} a_{\varphi(b)}^{\bullet}}
\end{aligned}
$$

$$
=\overline{a_{1} a_{2} \mathrm{~L} a_{\varphi(b)}},
$$

then we can obtain

$$
\frac{a}{b}=\frac{a k}{b k}=\frac{\overline{a_{1} a_{2} \mathrm{~L} a_{\varphi(b)}}}{10^{\varphi(b)}-1}=\overline{\dot{\bullet}_{1} a_{2} \mathrm{~L} \dot{a}_{\varphi(b)}} .
$$

Therefore $T(a / b)=\varphi(b)$.

In addition, since

$$
\varphi(b)=\varphi\left(\prod_{i=1}^{n} p_{i}^{k_{i}}\right)=\prod_{i=1}^{n} p_{i}^{k_{i}-1}\left(p_{i}-1\right)[4],
$$

so we have that

$$
T(a / b)=\varphi(b)=\prod_{i=1}^{n} p_{i}^{k_{i}-1}\left(p_{i}-1\right)
$$

Let $n_{0}$ be minimum positive integer such that

$$
10^{n} \equiv 1(\bmod b)
$$

then from Euler theorem, we further know that $n_{0} \mid \varphi(b)$.

By combining known conditions and Lemma 2(ii), we derived

$$
\underline{T}(a / b)=n_{0} .
$$

Consequently, we conclude that $\underline{T}(a / b) \mid T(a / b)$.

(ii) If $\underline{T}(a / b)=T(a / b)$, by Theorem 1 (i), we have $\underline{T}(a / b)=\varphi(b)$.

Supposing that there are $i, j(0 \leq i<j \leq \varphi(b)-1)$ such that

$$
10^{i} \equiv 10^{j}(\bmod b)
$$

we then have

$$
b \mid 10^{i}\left(10^{j-i}-1\right) .
$$

From $(10, b)=1$, we obtain $b \mid\left(10^{j-i}-1\right)$, that is, $10^{j-i} \equiv 1(\bmod b)$.

Note that Lemma 2(ii) and $1 \leq j-i<\varphi(b)$, obviously, this is in conflict with $\underline{T}(a / b)=\varphi(b)$. Therefore, we have $10^{i} \not \equiv 10^{j}(\bmod b)$, for any $i, j(0 \leq i<j \leq \varphi(b)-1)$.

Conversely, if $10^{i} \not \equiv 10^{j}(\bmod b)$, for any $i, j(0 \leq i$ $<j \leq \varphi(b)-1)$. Specially, by setting $i=0$, we have for any $j(1 \leq j \leq \varphi(b)-1)$ that 


$$
10^{j} \not \equiv 1(\bmod b),
$$

Moreover, from Euler theorem, we know that

$$
10^{\varphi(b)} \equiv 1(\bmod b)
$$

consequently, we have $\underline{T}(a / b)=\varphi(b)$.

Combining Theorm 1(i), we can obtain

$$
\underline{T}(a / b)=T(a / b) .
$$

The proof is completed.

Theorem 2 Let $a / b$ be an irreducible proper fraction, where

$$
b=2^{s} 5^{t} b_{1}, s \geq 0, t \geq 0, \quad s+t>0, b_{1}=\prod_{i=1}^{n} p_{i}^{k_{i}},
$$

$k_{i} \geq 1,\left(p_{i}, 10\right)=1$,

and $p_{i}$ is prime, then we have that

(i) $T(a / b)=\varphi\left(b_{1}\right)=\prod_{i=1}^{n} p_{i}^{k_{i}-1}\left(p_{i}-1\right)$ and

$\underline{T}(a / b) \mid T(a / b)$

(ii) $\underline{T}(a / b)=T(a / b)$ if and only if

$10^{i} \not \equiv 10^{j}\left(\bmod b_{1}\right)$, for any $i, j\left(0 \leq i<j \leq \varphi\left(b_{1}\right)-1\right)$.

Proof. From known conditions and Lemma 3, we know that $\underline{T}(a / b)$ is only related to $b_{1}$, therefore, Theorem 2 can be proved by the proof method of Theorem 1 . Here, we no longer prove the proposition.

Theorem 3 If $\left(b_{1}, b_{2}\right)=1$, then

$$
T\left(1 / b_{1}\right) T\left(1 / b_{2}\right)=T\left(1 / b_{1} b_{2}\right) .
$$

Proof. From $\left(b_{1}, b_{2}\right)=1$, we obtain

$$
\varphi\left(b_{1}\right) \varphi\left(b_{2}\right)=\varphi\left(b_{1} b_{2}\right)[4] .
$$

And by Thorem 1(i), we have that $T\left(1 / b_{1}\right)=\varphi\left(b_{1}\right)$, $T\left(1 / b_{2}\right)=\varphi\left(b_{2}\right)$ and $T\left(1 / b_{1} b_{2}\right)=\varphi\left(b_{1} b_{2}\right)$.

Consequently, we have

$$
T\left(1 / b_{1}\right) T\left(1 / b_{2}\right)=T\left(1 / b_{1} b_{2}\right) .
$$

The desired result follows.

According to the above conclusion, we can also obtain the following result.

Corollary If $\left(b_{i}, b_{j}\right)=1$, then

$$
\prod_{i=1}^{n} T\left(1 / b_{i}\right)=T\left(1 / \prod_{i=1}^{n} b_{i}\right) .
$$

Meanwhile, combine with the relevant data in table 1 and the above conclusions, we make the following guess

Guess If $\left(b_{i}, b_{j}\right)=1$, then

$$
\underline{T}\left(1 / \prod_{i=1}^{n} b_{i}\right) \mid \prod_{i=1}^{n} \underline{T}\left(1 / b_{i}\right) .
$$

\section{Conclusions And Prospect}

Through the above research, we have proposed the computation formulas of period of pure recurring decimal and mixed recurring decimal respectively, relation of the period and least positive period, and the necessary and sufficient condition that the period is eaqual to least positive period.But we think that the above conclusions are not perfect, in future studies, we will further weaken the sufficient and necessary conditions, and discuss the calculation formula of least positive period of recurring decimal and other beautiful properties.

\section{REFERENCES}

[1] Liying Sun and Xiaokui Ge, An application of the division algorithm and congruence to the periodic decimal, Journal Of Guangdong Education Institute, Vol 25(3),pp.31-33,2005.

[2] Limin Xue, Law of repetend of pure repeating decimal. Journal Of Weinan Teachers College, Vol 16(4),pp.92-94,2001.

[3] Dexin Zhang, Integer theory, Beijing:Science press, 1958.

[4] Chengdong Pan and Chengbiao Pan, Elementary number theory ,Beijing: Peking University Press, 1992.

[5] Yuejin Wang and Shanqi Pang, The new results on the fascinating properties of recurring decimals,Journal Of Henan Normal University(Natural Science), Vol 33(4),pp.25-26,2005.

[6] Shide Zhang,The fascinating properties of the recurring decimals,Journal Of Henan Normal University(Natural Science), Vol 30(3),pp.1114,2002 .

[7] Xiaosong Chen,The period and numerals sum of repeating decimal, Journal Of Yunnan University(Natural Science), Vol 29(3),pp.217222,2007.

[8] S.S.Wagner,Fun with repeating decimais, Mathematics Teacher, 3,pp.209-212,1979.

[9] K. Seydei, Cyciic fractions, Mathematics Teacher,11,pp.567-568,1979.

[10] M.W.Ec-Ker and S.Yates, Primes and cyciic fractions. Mathematics Teacher, 5,pp.327-390,1980. 